\title{
Efficacy and Safety of a Low-level Laser Device in the Treatment of Male and Female Pattern Hair Loss: A Multicenter, Randomized, Sham Device-controlled, Double-blind Study
}

\author{
Joaquin J. Jimenez $\cdot$ Tongyu C. Wikramanayake • \\ Wilma Bergfeld • Maria Hordinsky • Janet G. Hickman • \\ Michael R. Hamblin • Lawrence A. Schachner
}

Published online: 29 January 2014

(C) The Author(s) 2014. This article is published with open access at Springerlink.com

\begin{abstract}
Significance Male and female pattern hair loss are common, chronic dermatologic disorders with limited therapeutic options. In recent years, a number of commercial devices using low-level laser therapy have been promoted, but there have been little peer-reviewed data on their efficacy.

Objective To determine whether treatment with a lowlevel laser device, the US FDA-cleared HairMax Laser$\mathrm{comb}^{\circledR}$, increases terminal hair density in both men and women with pattern hair loss.

Methods Randomized, sham device-controlled, doubleblind clinical trials were conducted at multiple institutional and private practices. A total of 146 male and 188 female subjects with pattern hair loss were screened. A total of 128
\end{abstract}

Trial Registration: All trials were registered with http://www. clinicaltrials.gov. Trial \#1 (registration \#NCT00981461), "Treatment of Androgenetic Alopecia in Females, 9 Beam"; Trial \#2

(\#NCT01016964), "Treatment of Androgenetic Alopecia in Females, 12 Beam"; Trial \#3 (\#NCT00947505) and Trial \#4 (\#NCT00947219),

"Treatment of Androgenetic Alopecia in Males".

J. J. Jimenez ( $\square)$ · T. C. Wikramanayake $\cdot$ L. A. Schachner Department of Dermatology and Cutaneous Surgery, University of Miami Miller School of Medicine, Miami, FL 33136, USA

e-mail: j.jimenez@med.miami.edu

W. Bergfeld

Department of Dermatology, Cleveland Clinic Foundation, Cleveland, OH 44195, USA

M. Hordinsky

Department of Dermatology, University of Minnesota,

Minneapolis, MN 55455, USA

J. G. Hickman

The Education and Research Foundation, Inc., Lynchburg,

VA 24501, USA male and 141 female subjects were randomized to receive either a lasercomb (one of three models) or a sham device in concealed sealed packets, and were treated on the whole scalp three times a week for 26 weeks. Terminal hair density of the target area was evaluated at baseline and at 16- and 26-week follow-ups, and analyzed to determine whether the hypothesis formulated prior to data collection, that lasercomb treatment would increase terminal hair density, was correct. The site investigators and the subjects remained blinded to the type of device they dispensed/ received throughout the study. The evaluator of masked digital photographs was blinded to which trial arm the subject belonged.

Results Seventy-eight, 63, 49, and 79 subjects were randomized in four trials of 9-beam lasercomb treatment in female subjects, 12-beam lasercomb treatment in female subjects, 7-beam lasercomb treatment in male subjects, and 9- and 12-beam lasercomb treatment in male subjects, compared with the sham device, respectively. Nineteen female and 25 male subjects were lost to follow-up. Among the remaining 122 female and 103 male subjects in the

M. R. Hamblin

Wellman Center for Photomedicine, Massachusetts General

Hospital, Boston, MA 02114, USA

M. R. Hamblin

Department of Dermatology, Harvard Medical School, Boston, MA 02115, USA

M. R. Hamblin

Harvard-MIT Division of Health Sciences and Technology,

Cambridge, MA 02139, USA 
efficacy analysis, the mean terminal hair count at 26 weeks increased from baseline by 20.2, 20.6, 18.4, 20.9, and 25.7 per $\mathrm{cm}^{2}$ in 9-beam lasercomb-treated female subjects, 12-beam lasercomb-treated female subjects, 7-beam lasercomb-treated male subjects, and 9- and 12-beam lasercombtreated male subjects, respectively, compared with 2.8 $(p<0.0001), 3.0 \quad(p<0.0001), 1.6 \quad(p=0.0017), 9.4$ $(p=0.0249)$, and $9.4(p=0.0028)$ in sham-treated subjects $(95 \%$ confidence interval). The increase in terminal hair density was independent of the age and sex of the subject and the lasercomb model. Additionally, a higher percentage of lasercomb-treated subjects reported overall improvement of hair loss condition and thickness and fullness of hair in self-assessment, compared with sham-treated subjects. No serious adverse events were reported in any subject receiving the lasercomb in any of the four trials. Conclusions and relevance We observed a statistically significant difference in the increase in terminal hair density between lasercomb- and sham-treated subjects. No serious adverse events were reported. Our results suggest that low-level laser treatment may be an effective option to treat pattern hair loss in both men and women. Additional studies should be considered to determine the long-term effects of low-level laser treatment on hair growth and maintenance, and to optimize laser modality.

\section{Introduction}

Male and female pattern hair loss is a common, chronic dermatologic disorder. Male pattern hair loss (MPHL, or androgenetic alopecia, AGA) affects $50 \%$ of men by 50 years of age, and the frequency and severity increase with age [1]. MPHL is characterized by a dihydrotestosterone-dependent process with miniaturization of terminal hair follicles (HFs) into vellus HFs [2]. The frequency and severity of female pattern hair loss (FPHL) also increase with age, with a prevalence of over $50 \%$ in women over the age of 80 years [3]. While the role of androgens in all cases of FPHL is less certain, FPHL also undergoes follicular miniaturization [1]. Current medical treatments for pattern hair loss include topical minoxidil (available in $2 \%$ and $5 \%$ solutions or $5 \%$ foam, and sometimes combined with other active ingredients such as tretinoin), finasteride, dutasteride (US FDA approved for the treatment of benign prostatic hyperplasia, and prescribed off-label for treatment of MPHL), topical ketoconazole, anti-androgens and estrogens (for FPHL), and follicular unit transplantation [4]. In addition, there are numerous oral supplements and topical treatments claimed to have hair growth-promoting or anti-hair loss effects that are marketed directly to the consumers, without independent data supporting the claims.
In recent years, low-level laser/light therapy (LLLT), or photobiomodulation or photobiostimulation, has been promoted to prevent hair loss and stimulate hair growth in both MPHL and FPHL. There have been a number of commercially available devices designed for home use (daily or several times a week), and they are relatively inexpensive compared with current medical treatment and hair transplantation surgery. However, there have been few peerreviewed data on efficacy [5]. In one published study, only seven subjects with pattern hair loss (six female subjects and one male subject) were evaluated upon treatment with a laser "hood" [6]. The study was not sham device-controlled and the results did not reach statistical significance. A more recent, randomized, double-blind, sham device-controlled trial found "TOPHAT655" (a helmet-like device with lasers and light-emitting diodes) treatment to increase terminal hair count in pattern hair loss, but only male subjects were included in the trial [7]. To date, the most comprehensive published study is a randomized, double-blind, sham devicecontrolled clinical trial of 110 male subjects showing that the HairMax Lasercomb ${ }^{\circledR}$ (Lexington International, LLC., Boca Raton, FL, USA), FDA-cleared to treat pattern hair loss in male subjects at the time, was effective in increasing terminal hair density after 26 weeks of treatment [8]. The device has since been approved for treating FPHL, though there has been only one published study supporting the efficacy, with limitations [9]. In this study, only seven female subjects were included. They were given a lasercomb to use for 6 months, and the terminal hair count was compared between baseline and at the end of the study. The FDA considered the LaserComb ${ }^{\circledR}$ a medical device of "moderate risk", therefore it only screened for safety, not efficacy.

Given the prevalence of MPHL and FPHL, their limited medical treatment and the high costs of hair transplantation, and the ready availability and user friendliness of LLLT home devices, it is important to determine whether LLLT can provide an effective alternative for pattern hair loss, especially FPHL, for which no randomized, controlled trials have been published. The objective of this study was to determine the efficacy of LaserComb ${ }^{\circledR}$ treatment of pattern hair loss in both male and female subjects, in four randomized, multicenter, sham device-controlled, double-blind prospective trials. A total of 122 female and 103 male subjects were included in the efficacy analysis after 26 weeks of treatment, and three lasercomb models were evaluated.

\section{Methods}

\subsection{Patient Enrollment}

The study protocol was evaluated under Good Clinical Practice guidelines and approved by the authors' 
Institutional Review Boards (IRBs) or the Chesapeake Research Review, Inc. All trials were registered with http:// www.clinicaltrials.gov. Prior to participation in the trials, each subject provided a written informed consent. Participants received free evaluations at baseline and at followups. They were compensated for each visit and were given a lasercomb at the end of the study (26-week visit). Subject screening, recruitment, and follow-up were carried out at multiple study sites: Trial \#1 (registration \#NCT00981461), "Treatment of Androgenetic Alopecia in Females, 9 Beam": International Dermatology Research, Inc. (Miami, FL, USA), The Education \& Research Foundation, Inc. (Lynchburg, VA, USA); Trial \#2 (\#NCT01016964), "Treatment of Androgenetic Alopecia in Females, 12 Beam": The Cleveland Clinic Foundation (Cleveland, OH, USA), University of Minnesota (Minneapolis, MN, USA), University of Miami Miller School of Medicine (Miami, FL, USA); Trial \#3 (\#NCT00947505) and Trial \#4 (\#NCT00947219), "Treatment of Androgenetic Alopecia in Males": Dermatology Consulting Services (High Point, NC, USA); Trials \#1, \#3, and \#4: DermResearch, Inc. (Austin, TX, USA), Skin Laser and Surgery Specialist (Hillsborough, NJ, USA), and Palm Beach Research Center (West Palm Beach, FL, USA). Full trial protocol is available upon request.

\subsubsection{Study Inclusion/Exclusion Criteria}

To be included in the trials, subjects must have been healthy, 25-60 years of age, with active androgenetic hair loss (Norwood-Hamilton classification of IIa-V for male subjects [10] and Ludwig/Savin classification of I-4, II-1, II-2, or frontal for female subjects) [11-13] and have Fitzpatrick skin type I-IV [14]. Race/ethnicity information was collected. Subjects must not have taken or used the following medications within 6 months prior to screening: minoxidil, finasteride (or any other $5 \alpha$-reductase inhibitors), medications with anti-androgenic properties (e.g., cyproterone acetate, spironolactone, ketoconazole, flutamide, bicalutamide), topical estrogen, progesterone, tamoxifen, anabolic steroids, medication that can potentially cause hypertrichosis (e.g., cyclosporine, diazoxide, phenytoin, psoralens), oral glucocorticoids (inhaled glucocorticoids were permitted), lithium, phenothiazines, or other medications at the discretion of the investigators. Other excluded medications were phytotherapy (e.g., saw palmetto) within 8 weeks, isotretinoin within the past year, and anticoagulation use [other than aspirin $(<325 \mathrm{mg}$ every day, which was stable for 3 months)]. Subjects were excluded if they had malignancy in the target area within 5 years, active infection on the scalp, chronic dermatologic conditions (e.g., eczema, psoriasis, infection) of the scalp other than pattern hair loss, a history of poor wound healing or keloid formation, a history of thyroid or other medical condition that might influence hair growth and loss; human immunodeficiency virus infection, possession of a pacemaker, defibrillator, or other active implantable device; a history of drug and/or alcohol abuse within the past 12 months; or any other medical conditions at the discretion of the investigators. Pregnant female subjects or female subjects planning on becoming pregnant during the duration of the study were excluded. Subjects with a history of photosensitivity to laser light, hair transplantation, scalp reduction, radiation to the scalp or chemotherapy within the past year, current hair weave or tattooing, as well as subjects with hair shorter than one-half inch or with light-blonde hair were also excluded.

\subsection{The Lasercomb and Sham Devices}

Three different lasercomb configurations were evaluated for similar laser dose rates. These models were designed to meet varying marketing demands, and the FDA required clinical studies on each model to ensure consistency of results. The 7- and 9-beam lasercombs (HairMax Laser$\mathrm{Comb}^{\circledR}$, Lexington International, LLC) emit 7 or 9 red laser beams (beam diameter $<5 \mathrm{~mm}$ ) at a wavelength of $655 \mathrm{~nm}( \pm 5 \%)$. The 12-beam dual model emits 6 beams at a wavelength of $635 \mathrm{~nm}( \pm 5 \%)$ and 6 beams at $655 \mathrm{~nm}$ $( \pm 5 \%)$. The lasers for each device were identical in power output, and the treatment time was adjusted for similar laser dose rates: $15 \mathrm{~min}$ for the 7-beam model, $11 \mathrm{~min}$ for the 9-beam model, and $8 \mathrm{~min}$ for the 12-beam model. Two sham devices that emitted white light from light-emitting diode bulbs had identical appearance as the 7- and 9-beam lasercombs, and were used as controls for the 7-, and 9- or 12-beam lasercombs, respectively.

\subsection{Study Design}

Four multicenter prospective trials were designed, to be randomized, sham controlled, and double blind. In Trials $\# 1$ and \#2, subjects with FPHL used a 9-beam (\#1) or a dual 12-beam (\#2) lasercomb and sham device. In Trials \#3 and \#4, subjects with MPHL used a 7- $(\# 3)$ or a 9- or 12-beam (\#4) lasercomb and sham device.

Each study protocol was approved by institutional or the Chesapeake IRB. Each Clinical Study Sponsor confirmed performance in compliance with Good Clinical Practice (GCP, as defined in CPMP/ICH/135/95), the Declaration of Helsinki (with amendments), and local legal and regulatory requirements. Lexington International LLC, as a company, is and has been compliant and certified to ISO9001 and ISO13485 Quality Standards. Lexington's Clinical Study Practices have been audited by the FDA and have confirmed to be in compliance with the FDA's GCP. All 
studies were managed and audited by Palm Beach CRO (Clinical Research Organization) and validated to be in compliance with the approved protocol.

For subjects who met the inclusion and exclusion criteria, at the baseline visit, a "target site" in the affected scalp area was chosen using a $25 \mathrm{~mm} \times 25 \mathrm{~mm}$ plastic template, and hair within this target site $(25 \mathrm{~mm} \times 25 \mathrm{~mm})$ was clipped. The target site was then marked with a semipermanent tattoo using a professional tattooing machine (K.P. Permanent Make-Up, Inc., Pomona, CA, USA), and photographed.

Each subject was then provided with either a lasercomb or a sham device. Randomization was generated by Eugene R. Heyman (http://www.erhstats.com) using the SAS PROC RAND method. For the 9- and 12-beam trial in male subjects (\#4), randomization was generated 1:1:1 with a block size of 3. For all other trials, randomization was 2:1 with a block size of 3 . The lasercomb and sham devices, along with instructions, were provided to the site investigators in sealed, sequentially numbered opaque packets in a blinded manner, and were dispensed sequentially. Both the site investigators and the subjects remained blinded to the type of device they dispensed/received throughout the study.

The subjects were instructed to apply the device three times per week, with the beam on, to their entire scalp; the duration of treatment specific for each device and their respective sham control was included in the sealed packet (15 min for the 7-beam model, $11 \mathrm{~min}$ for the 9-beam model, and $8 \mathrm{~min}$ for the 12-beam model). Each subject was required to keep a diary of usage, which was reviewed by the site investigator at the time of office visits. The study duration was 26 weeks, with clinical monitoring visits at 8,16 , and 26 weeks. Dermatology scalp assessment, safety assessment, global and macro digital imaging after hair clipping, and computer-aided hair counts of the target sites were performed by blinded investigators at weeks 16 and 26, and compared with baseline.

\subsubsection{Efficacy Evaluation}

Change of terminal hair density (hair count $/ \mathrm{cm}^{2}$ ) at 26 weeks from baseline was used as the endpoint to evaluate the efficacy of lasercomb treatment in male and female subjects with pattern hair loss. The Canfield Epilume System was used for digital imaging of the target sites at baseline and at weeks 16 and 26. All macro photographs, with a $10-\mathrm{mm}$ scale bar divided in $0.1-\mathrm{mm}$ increments, were labeled only by subject number and uploaded to an online database. An independent evaluator not connected to the clinical trials analyzed the uploaded images and performed computer-assisted hair counts, using the TrichoScience software (Tricholog, Moscow, Russia). The evaluator was a hair transplant surgeon with 20 years of experience in evaluation of hair counts, and was blinded to which trial arm the subject belonged, as well as which images were from baseline and which were from follow-up. Subjects also filled out questionnaires for self-assessment of overall improvement of hair loss condition and thickness and fullness of hair at the 16- and 26-week visits.

\subsection{Statistical Analysis}

Based on previous testing data on lasercomb use, change in terminal hair count from baseline to study endpoint was found to be a mean increase of just under 30 hairs $/ \mathrm{cm}^{2}$ with a standard deviation of 18.6 hairs $/ \mathrm{cm}^{2}$. For the sample size calculation, the assumed standard deviation was 20 hairs/ $\mathrm{cm}^{2}$ and the treatment difference was assumed to be 17 hairs $/ \mathrm{cm}^{2}$. Each trial had a planned enrollment of $60 \mathrm{sub}-$ jects in a 2:1 allocation of lasercomb:sham device to achieve at least $80 \%$ power while allowing a $10 \%$ dropout rate. In Trials \#1-3, subjects were randomized to a 2:1 allocation of the lasercomb:sham device. In Trial \#4, subjects were randomized in a 1:1:1 allocation of the 9-beam:12-beam:sham device. For subject enrollment, continuous variables (e.g., age) were analyzed with a oneway analysis of variance and categorical variables with the Fisher's exact test.

The primary efficacy endpoint was the change in terminal hair density within the target area at 26 weeks from baseline, assessed in all subjects with baseline and at least one post-randomization efficacy evaluation. The lasercomb-treated group was compared with the sham device group using least squares mean with two-sided at a $5 \%$ level of significance. The primary analysis of efficacy was an analysis of co-variance, which modeled terminal hair density as a function of treatment group, study center, age (as a continuous variable), and Fitzpatrick skin type (as a categorical variable). The secondary efficacy endpoint was the categorical change in terminal hair density from baseline, analyzed using the Cochran-Mantel-Haenszel row mean score test with integer scores stratified by study site. Cochran's $Q$ test was performed to analyze the homogeneity of results across genders, all trials, and all lasercomb models. Subject self-assessment was also evaluated using the Cochran-Mantel-Haenszel row mean score test with integer scores stratified by site. The DerSimonian-Laird approach was used to perform the meta-analysis homogeneity assessment. All statistical analyses were contracted to Stat-Tech Services, LLC (Chapel Hill, NC, USA). For evaluation of safety, adverse events were summarized and each event was evaluated for frequency. 


\section{Results}

\subsection{Study Population}

A total of 188 female and 146 male subjects were screened, and 141 female and 128 male subjects were randomized to receive the lasercomb or sham device. Of these subjects, 19 female and 25 male subjects were lost to follow-up, leaving 122 female and 103 male subjects completing at least one follow-up. Sixty-five and 57 subjects (122 total) were included in the efficacy evaluation for Trials \#1 and \#2 (the female trials evaluating the 9-beam and dual 12-beam lasercomb, respectively) (Fig. 1; Table 1), and 38 and 65 subjects (103 total) were included in the efficacy evaluation for Trials \#3 and \#4 (the male trials evaluating the 7- and the 9- or 12-beam lasercomb, respectively) (Fig. 1; Table 2). There were no statistically significant differences in demographic characteristics or hair loss features between the lasercomb and sham group in any of the four trials at baseline (Tables 1 and 2). The age of the subjects was 25-61 years, and $94.7 \%$ were Caucasian. The last follow-up was conducted after 26 weeks of treatment, an accepted standard for clinical trials on hair growth.

\subsection{Analysis of Efficacy}

The trials were designed to be randomized and double blind. Data from different study sites were pooled for statistical analysis. All the randomized subjects who had a baseline and at least one post-randomization evaluation were included in the efficacy analysis (Fig. 1). To account for dropouts thereafter, all data are presented in last observation carried forward for the analysis of covariance for Trials \#1 and \#4.

\subsubsection{Primary Efficacy Analysis}

In Trial \#1, a significant difference in terminal hair density change from baseline was observed between the 9-beam lasercomb- and sham-treated female subjects at 26 weeks $(p<0.0001) \quad$ (Fig. 2a). The lasercomb-treated subjects showed a much higher increase in terminal hair density compared with sham-treated subjects, with a mean of 20.2 ( \pm 11.2 standard deviation $[\mathrm{SD}])$ versus $2.8( \pm 16.5 \mathrm{SD})$ per $\mathrm{cm}^{2}$ (Fig. 2a). Similar improvement in terminal hair density was observed with the 12-beam lasercomb treatment in Trial \#2 (Fig. 2b). The lasercomb-treated female subjects
Fig. 1 Profile of the four randomized, sham-controlled trials of lasercomb treatment of male and female pattern hair loss. Dates of recruitments are indicated

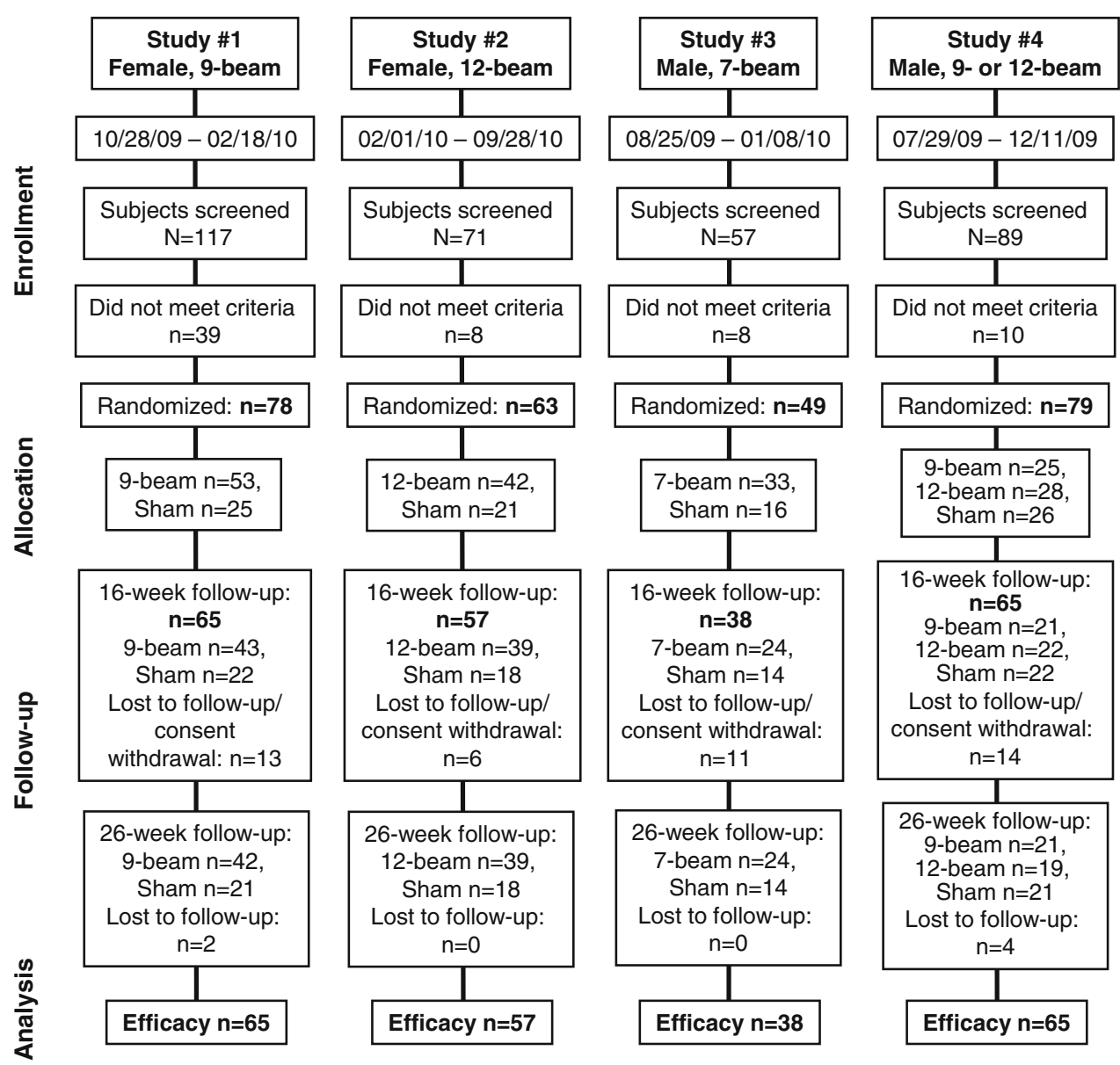


Table 1 Demographic characteristics of female subjects at baseline for the 9- and 12-beam lasercomb trials

\begin{tabular}{|c|c|c|c|c|c|c|}
\hline & \multicolumn{3}{|l|}{ Trial \#1 $(n=65)$} & \multicolumn{3}{|l|}{ Trial \#2 $(n=57)$} \\
\hline & 9-beam lasercomb & Sham & $p$ value & 12-beam lasercomb & Sham & $p$ value \\
\hline Number of subjects & 43 & 22 & & 39 & 18 & \\
\hline Age (years) & & & 0.8261 & & & 0.9102 \\
\hline Mean age (SD) & $49.3(9.1)$ & $49.8(7.3)$ & & $48.7(10.2)$ & $49.1(8.3)$ & \\
\hline Median age & 52 & 49 & & 50 & 49 & \\
\hline Range & $29-60$ & $37-60$ & & $26-61$ & $33-60$ & \\
\hline Race, $n(\%)$ & & & 1.0000 & & & 1.0000 \\
\hline Caucasian & $39(90.7 \%)$ & $20(90.9 \%)$ & & $37(94.9 \%)$ & $18(100.0 \%)$ & \\
\hline African American & $1(2.3 \%)$ & $0(0 \%)$ & & $1(2.6 \%)$ & $0(0 \%)$ & \\
\hline Native American & $0(0 \%)$ & $0(0 \%)$ & & $0(0 \%)$ & $0(0 \%)$ & \\
\hline Alaska Native & $0(0 \%)$ & $0(0 \%)$ & & $0(0 \%)$ & $0(0 \%)$ & \\
\hline Asia/Pacific Islander & $2(4.7 \%)$ & $1(4.5 \%)$ & & $1(2.6 \%)$ & $0(0 \%)$ & \\
\hline Other & $1(2.3 \%)$ & $1(4.5 \%)$ & & $0(0 \%)$ & $0(0 \%)$ & \\
\hline Ethnicity, $n(\%)$ & & & 0.2773 & & & 1.0000 \\
\hline Hispanic or Latino & $13(30.2 \%)$ & $10(45.5 \%)$ & & $10(25.6 \%)$ & $4(22.2 \%)$ & \\
\hline Not Hispanic or Latino & $30(69.8 \%)$ & $12(54.5 \%)$ & & $29(74.4 \%)$ & $14(77.8 \%)$ & \\
\hline Ludwig/Savin classification, $n(\%)$ & & & 0.6513 & & & 0.2926 \\
\hline $\mathrm{I}-4$ & $12(27.9 \%)$ & $3(13.6 \%)$ & & $21(53.8 \%)$ & $6(33.3 \%)$ & \\
\hline II-1 & $11(25.6 \%)$ & $7(31.8 \%)$ & & $11(28.2 \%)$ & $6(33.3 \%)$ & \\
\hline II-2 & $15(34.9 \%)$ & $9(40.9 \%)$ & & $6(15.4 \%)$ & $4(22.2 \%)$ & \\
\hline Frontal & $5(11.6 \%)$ & $3(13.6 \%)$ & & $1(2.6 \%)$ & $2(11.1 \%)$ & \\
\hline Fitzpatrick skin type, $n(\%)$ & & & 1.0000 & & & 0.7606 \\
\hline I & $0(0 \%)$ & $0(0 \%)$ & & $2(5.1 \%)$ & $0(0 \%)$ & \\
\hline II & $15(34.9 \%)$ & $7(31.8 \%)$ & & $11(28.2 \%)$ & $4(22.2 \%)$ & \\
\hline III & $20(46.5 \%)$ & $11(50.0 \%)$ & & $14(35.9 \%)$ & $9(50.0 \%)$ & \\
\hline IV & $8(18.6 \%)$ & $4(18.2 \%)$ & & $12(30.8 \%)$ & $5(27.8 \%)$ & \\
\hline Mean baseline hair count ${ }^{\mathrm{a}}(\mathrm{SD})$ & $162.6(46.2)$ & $155.7(43.5)$ & & $142.2(40.5)$ & $168.4(41.1)$ & \\
\hline
\end{tabular}

${ }^{\text {a }}$ Number of terminal hairs per $\mathrm{cm}^{2}$ in the target area

$S D$ standard deviation

had a mean increase in terminal hair density of $20.6( \pm 11.6$ SD) compared with $3.0( \pm 9.3 \mathrm{SD})$ for the sham group (Fig. 2b). Overall, primary efficacy analysis showed the difference in terminal hair density change at 26 weeks from baseline between lasercomb and sham treatment was highly significant $(p<0.0001)$ in both female trials (Fig. 2a, b). Similarly, statistically significant improvement was observed with lasercomb treatment compared with sham treatment in both male trials (Trial \#3, 7-beam lasercomb vs. sham, $p=0.0017$, Fig. 2c; Trial \#4, 9- and 12-beam lasercombs vs. sham, $p=0.0249$ and $p=0.0028$, for the 9- and 12-beam lasercombs, respectively, Fig. 2d).

\subsubsection{Secondary Efficacy Analyses}

Secondary efficacy analyses included categorical summaries and covariate analyses of changes in terminal hair density from baseline. In Trial \#1, 41 of 43 (95\%) of the 9-beam lasercomb-treated female subjects had hair density improvement of $>5$ hairs $/ \mathrm{cm}^{2}$ at 26 weeks while only 7 of $22(32 \%)$ sham-treated female subjects did $(p<0.0001)$ (Fig. 2e). Additionally, none of the 43 lasercomb-treated subjects showed decreased hair density as opposed to 11 of $22(50 \%)$ sham-treated subjects (Fig. 2e). Analysis of data collected at 16 weeks revealed similar results (data not shown). In Trial \#2, 37 of 39 (95\%) of the 12-beam lasercomb-treated female subjects had hair density improvement of $>5$ hairs $/ \mathrm{cm}^{2}$ while only 6 of $18(33 \%)$ sham-treated female subjects did $(p<0.0001)$ (Fig. 2f). Although 7 of 18 (39\%) sham-treated subjects showed decreased hair density, only 1 of 39 (3\%) lasercombtreated subjects did (Fig. 2f).

In Trial \#3, 20 of 24 (83\%) of the 7-beam lasercombtreated male subjects had hair density improvement of $>5$ hairs $/ \mathrm{cm}^{2}$, while only 6 of $14(43 \%)$ sham-treated male subjects did ( $p=0.0033$ ) (Fig. $2 \mathrm{~g}$ ). Additionally, only 2 of the $24(8 \%)$ lasercomb-treated male subjects showed 
Table 2 Demographic characteristics of male subjects at baseline for the 7-, 9-, or 12-beam lasercomb trials

\begin{tabular}{|c|c|c|c|c|c|c|c|}
\hline & \multicolumn{3}{|c|}{ Trial \#3 $(n=38)$} & \multicolumn{4}{|c|}{ Trial \#4 $(n=65)$} \\
\hline & $\begin{array}{l}\text { 7-beam } \\
\text { lasercomb }\end{array}$ & Sham & $p$ value & $\begin{array}{l}\text { 9-beam } \\
\text { lasercomb }\end{array}$ & $\begin{array}{l}\text { 12-beam } \\
\text { lasercomb }\end{array}$ & Sham & $p$ value \\
\hline Number of subjects & 24 & 14 & & 21 & 22 & 22 & \\
\hline Age (years) & & & 0.0327 & & & & 0.7100 \\
\hline Mean age (SD) & $47.8(9.0)$ & $40.9(9.5)$ & & $45.6(9.3)$ & $47.9(9.6)$ & $45.9(10.4)$ & \\
\hline Median age & 48 & 41.5 & & 50 & 50.5 & 47 & \\
\hline Range & $26-59$ & $25-55$ & & $26-58$ & $26-59$ & $30-61$ & \\
\hline Race, $n(\%)$ & & & 1.0000 & & & & 1.0000 \\
\hline Caucasian & $23(95.8 \%)$ & $13(92.9 \%)$ & & $21(100.0 \%)$ & $21(95.5 \%)$ & $21(95.5 \%)$ & \\
\hline African American & $0(0 \%)$ & $0(0 \%)$ & & $0(0 \%)$ & $0(0 \%)$ & $0(0 \%)$ & \\
\hline Native American & $0(0 \%)$ & $0(0 \%)$ & & $0(0 \%)$ & $0(0 \%)$ & $0(0 \%)$ & \\
\hline Alaska native & $0(0 \%)$ & $0(0 \%)$ & & $0(0 \%)$ & $0(0 \%)$ & $0(0 \%)$ & \\
\hline Asia/Pacific islander & $0(0 \%)$ & $0(0 \%)$ & & $0(0 \%)$ & $0(0 \%)$ & $1(4.5 \%)$ & \\
\hline Other & $1(4.2 \%)$ & $1(7.1 \%)$ & & $0(0 \%)$ & $1(4.5 \%)$ & $0(0 \%)$ & \\
\hline Ethnicity, $n(\%)$ & & & 0.6497 & & & & 0.041 \\
\hline Hispanic or Latino & $3(12.5 \%)$ & $3(21.4 \%)$ & & $4(19.0 \%)$ & $1(4.5 \%)$ & $0(0 \%)$ & \\
\hline Not Hispanic or Latino & $21(87.5 \%)$ & $11(78.6 \%)$ & & $17(81.0 \%)$ & $21(95.5 \%)$ & $22(100.0 \%)$ & \\
\hline $\begin{array}{l}\text { Norwood-Hamilton classification, } \\
n(\%)\end{array}$ & & & 0.9130 & & & & 1.0000 \\
\hline II & $0(0 \%)$ & $0(0 \%)$ & & $0(0 \%)$ & $0(0 \%)$ & $1(4.5 \%)$ & \\
\hline III & $10(41.7 \%)$ & $5(35.7 \%)$ & & $10(47.6 \%)$ & $10(45.5 \%)$ & $10(45.5 \%)$ & \\
\hline IV & $9(37.5 \%)$ & $5(35.7 \%)$ & & $8(38.1 \%)$ & $9(40.9 \%)$ & $7(31.8 \%)$ & \\
\hline $\mathrm{V}$ & $5(20.8 \%)$ & $4(28.6 \%)$ & & $3(14.3 \%)$ & $3(13.6 \%)$ & $4(18.2 \%)$ & \\
\hline Fitzpatrick skin type (\%) & & & 0.7904 & & & & 0.998 \\
\hline I & $1(4.2 \%)$ & $0(0 \%)$ & & $3(14.3 \%)$ & $2(9.1 \%)$ & $2(9.1 \%)$ & \\
\hline II & $3(12.5 \%)$ & $3(21.4 \%)$ & & $9(42.9 \%)$ & $10(45.5 \%)$ & $9(40.9 \%)$ & \\
\hline III & $12(50.0 \%)$ & $5(35.7 \%)$ & & $7(33.3 \%)$ & $8(36.4 \%)$ & $9(40.9 \%)$ & \\
\hline IV & $8(33.3 \%)$ & $6(42.9 \%)$ & & $2(9.5 \%)$ & $2(9.1 \%)$ & $2(9.1 \%)$ & \\
\hline Mean baseline hair count ${ }^{\mathrm{a}}$ (SD) & $211.5(54.0)$ & $216.6(34.8)$ & & $163.3(69.4)$ & $151.5(42.4)$ & $171.4(62.3)$ & \\
\hline
\end{tabular}

${ }^{a}$ Number of terminal hairs per $\mathrm{cm}^{2}$ in the target area

$S D$ standard deviation

decreased hair density, while 6 of $14(43 \%)$ sham-treated subjects did (Fig. 2g). In Trial \#4, lasercomb-treated male subjects showed a higher percentage for hair density improvement of $>5$ hairs $/ \mathrm{cm}^{2}$ with either lasercomb model ( $86 \%$ for the 9-beam model and $82 \%$ for the 12-beam model) than the sham-treated subjects (59\%) (Fig. 2h). Whereas 9 of $22(41 \%)$ sham-treated subjects showed decreased hair density, only 3 of 21 (14\%) 9-beam lasercomb-treated subjects and 4 of $22(18 \%)$ 12-beam lasercomb-treated subjects did ( $p=0.0033)$ (Fig. 2h).

Overall, we observed significant categorical improvement in terminal hair density with lasercomb treatment versus control (Fig. 2e-h). Taken together, all four trials using three different lasercomb models in both male and female subjects showed improvement in terminal hair density that was highly statistically significant, as well as categorical improvement, with lasercomb treatment compared with sham treatment at 26 weeks.

\subsubsection{Subject Self-Assessment}

A higher percentage of lasercomb-treated subjects reported overall improvement of hair loss condition and thickness and fullness of hair in self-assessment, compared with sham-treated subjects (Table 3). In Trial \#1, statistical significance was reached for the assessment of both the overall improvement of hair loss condition and thickness and fullness of hair. Results in Trial \#2 did not reach statistical significance. In the pooled male subject trials, assessment of the thickness and fullness of hair reached statistical significance, but not the overall improvement of hair loss condition (Table 3). 

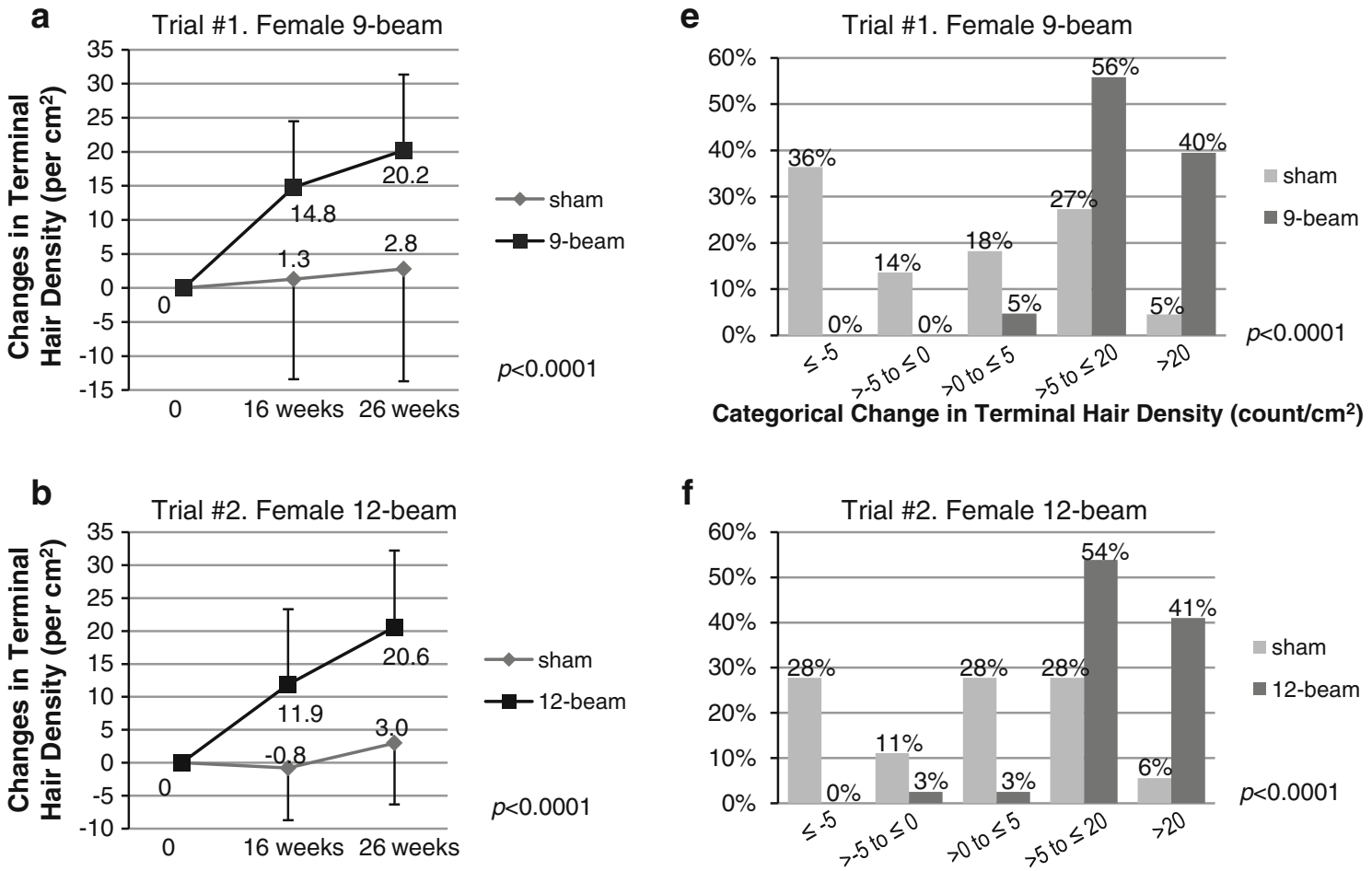

Categorical Change in Terminal Hair Density (count/cm²)

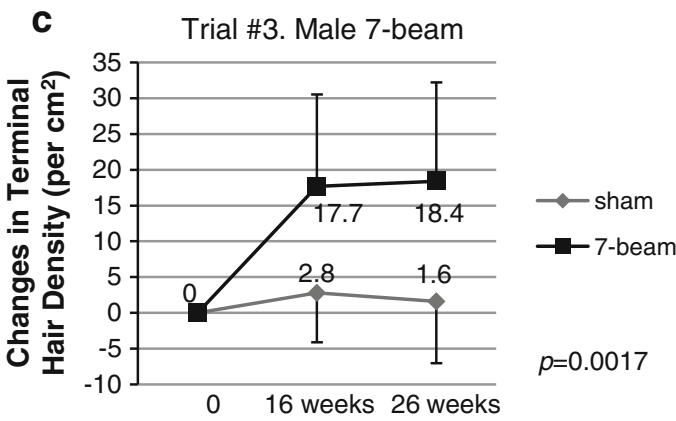

g

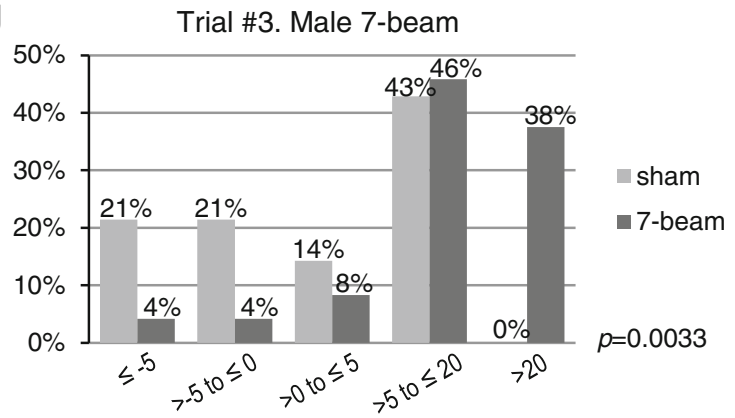

Categorical Change in Terminal Hair Density (count/cm²)
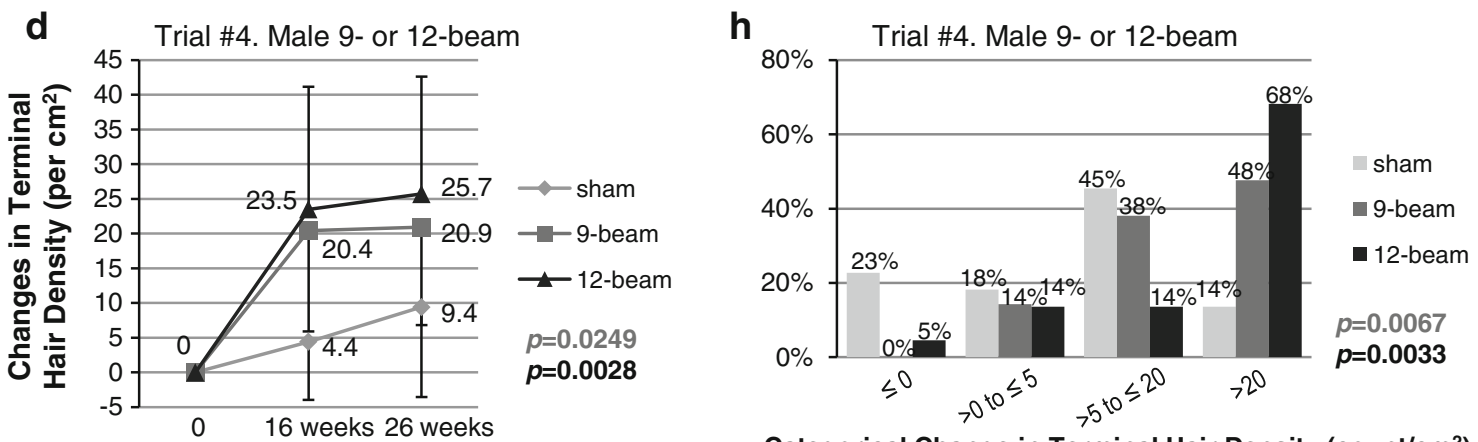

Categorical Change in Terminal Hair Density (count/cm²)

Fig. 2 a-d Mean changes in terminal hair density (count per $\mathrm{cm}^{2}$ ) from baseline in subjects treated with the lasercomb or sham device. Bars indicate standard deviation. $\mathbf{e}-\mathbf{h}$ Categorical changes in terminal

hair density (count per $\mathrm{cm}^{2}$ ) from baseline to 26 weeks in subjects treated with the lasercomb or sham device. Shown are $p$ values at 26 weeks 


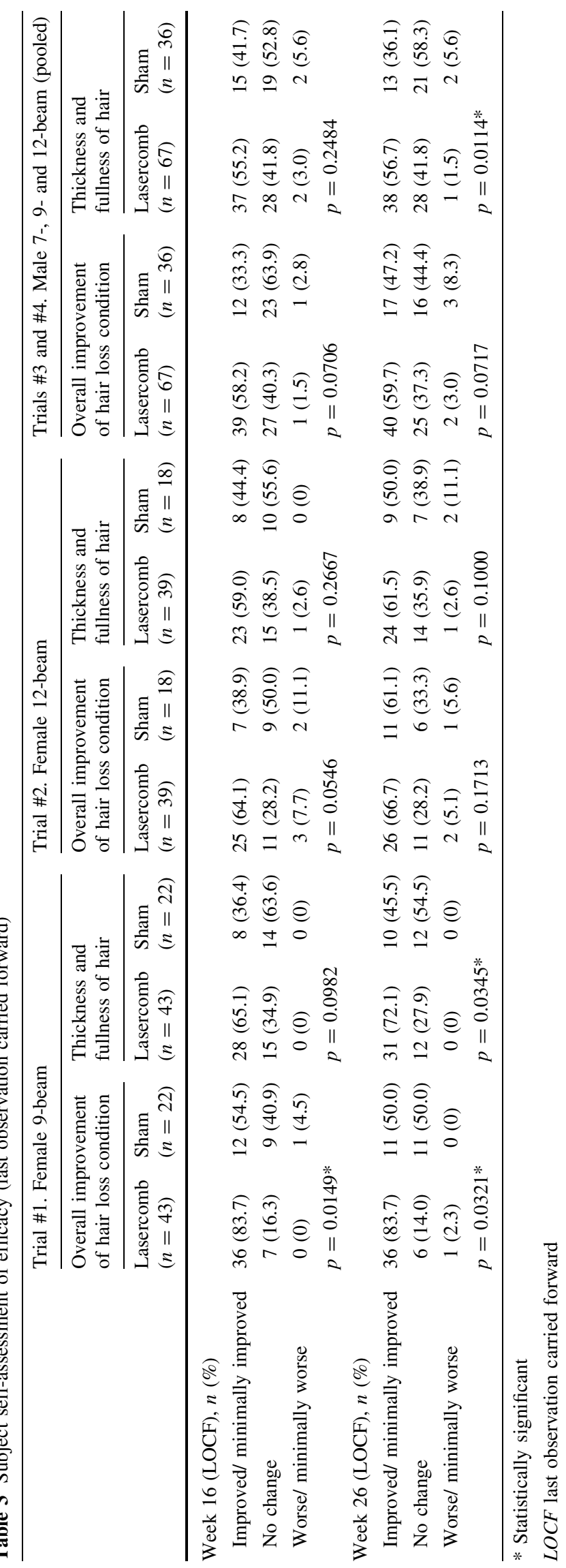


Fig. 3 Male and female pattern hair loss before and after lasercomb treatment. Global photographs of a female subject, at baseline (a) and after 26 weeks (b) of the 12-beam lasercomb treatment.

Macrophotographs of a male subject, at baseline (c) and after 26 weeks (d) of the 9-beam lasercomb treatment. Increased hair count through conversion of vellus or intermediate follicles to active follicles producing terminal hair (ovals) or resting telogen to active anagen follicles (rectangles) is highlighted
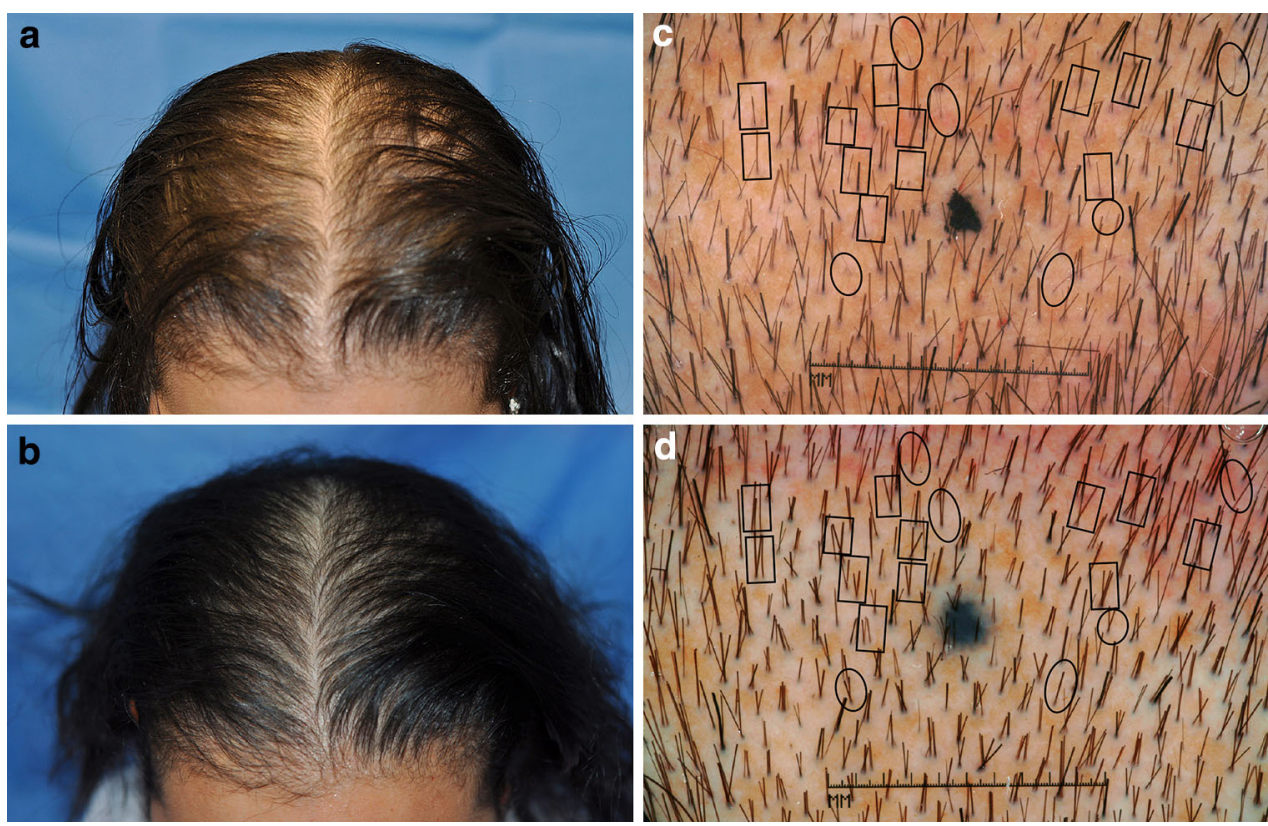

\subsubsection{Meta-Analysis of the Effects of Lasercomb Model, Study Duration, and Gender}

Meta-analyses were conducted to provide an overall assessment of the individual study results. The overall results showed the least squares mean difference of change in terminal hair density of 15.27 (standard error 1.781) at 26 weeks from baseline between lasercomb- and sham treated subjects, which was highly statistically significant $(p<0.0001)$. The homogeneity assessment results were non-significant $(p=0.6188)$. These results indicated that compared with sham treatment, lasercomb treatment resulted in a statistically significant increase in terminal hair density across the trials, independent of the lasercomb model (7- and 9-beam $655 \mathrm{~nm} \pm 5 \%$ laser and 12-beam $635 \mathrm{~nm}$ and $655 \mathrm{~nm} \pm 5 \%$ laser) and the sex of the subject.

Before and after global photographs (Fig. 3a, b) and macrophotographs (Fig. 3c, d) demonstrated increases in terminal hair density, most likely through the conversion of vellus or intermediate follicles to terminal follicles or from resting telogen follicles to active anagen follicles.

In summary, efficacy analysis showed a statistically significant increase in terminal hair density after 26 weeks of lasercomb treatment compared with sham treatment. The mean increase in terminal hair density was higher (statistically significant) in lasercomb-treated subjects than in sham-treated subjects. Additionally, a higher percentage of lasercomb-treated subjects showed categorical hair density improvement $\left(>5\right.$ hairs $\left./ \mathrm{cm}^{2}\right)$ at 26 weeks, compared with sham-treated subjects. Such improvement was observed in all four trials, and independent of the sex and age of the subject, and independent of the lasercomb model when similar laser dose rates were delivered. A higher percentage of lasercomb-treated subjects reported overall improvement of hair loss condition and thickness and fullness of hair in self-assessment, though the results did not always reach statistical significance.

\subsection{Safety and Tolerability}

No serious adverse events were reported in any subject receiving the lasercomb in any of the four trials. Reported lasercomb-related adverse events included dry skin $(5.1 \%)$, pruritus $(2.5 \%)$, scalp tenderness $(1.3 \%)$, irritation $(1.3 \%)$, and a warm sensation at the site $(1.3 \%)$. No subjects experienced an adverse event that resulted in the discontinuation of the study device, or interruption of the study. No adverse events had an impact on the study device use. There were no significant differences in active device adverse events as recorded by device type.

\section{Discussion}

Pattern hair loss may affect up to $70 \%$ of men and $50 \%$ of women at some point in their lifetime [3,4]. There has been an urgent need to determine whether LLLT home devices, which have been widely promoted for the treatment of MPHL and FPHL despite few randomized, controlled trials, can provide an effective alternative for patients with pattern hair loss, especially female patients. In this study, through four randomized, multicenter, sham device-controlled and double-blind clinical trials, we have shown that 26 weeks of treatment with the FDA-cleared HairMax LaserComb ${ }^{\circledR}$, compared with sham treatment, 
resulted in a statistically significant terminal hair density increase. Our results not only verified the effective treatment of MPHL reported previously [8], but also showed treatment efficacy in female subjects, and demonstrated that the treatment efficacy was independent of the laser configurations tested when similar laser dose rates were delivered. No serious adverse events were reported in any subject receiving lasercomb treatment in any of the four trials.

We have observed increased terminal hair density likely through both conversion of vellus or intermediate follicles to active follicles producing terminal hair and conversion of resting telogen follicles to active anagen follicles (Fig. 3c, d). The exact mechanisms of such conversions by LLLT remain unknown. Commonly used LLLT encompasses a wavelength of $500-1,100 \mathrm{~nm}$ and delivers fluences of $1-4 \mathrm{~J} / \mathrm{cm}^{2}$ with a power density of $3-90 \mathrm{~mW} / \mathrm{cm}^{2}$, and has demonstrated beneficial effects in various conditions including wound healing, joint pain relief, mucositis prevention and treatment, and skin conditions [15-22]. Based on anecdotal experience, LLLT of $650-900 \mathrm{~nm}$ wavelengths at $5 \mathrm{~mW}$ has been suggested to be an effective treatment option for male and female patients with pattern hair loss [23], though comprehensive studies evaluating laser modality are lacking. Whereas the exact mechanisms of hair growth stimulation by LLLT remain unknown, LLLT has been proposed to accelerate mitosis [24], and may stimulate HF stem cells or activate follicular keratinocytes. Additionally, laser light may alter cell metabolism through photodissociation of inhibitory nitric oxide from cytochrome c oxidase [25] (unit IV in the respiratory chain of mitochondria), causing increased ATP production and cellular activity [26]. Furthermore, resolution of inflammation may be one potential mechanism of hair growth stimulation by LLLT in AGA [27-32]. In vitro and in vivo trials of LLLT have shown decreased inflammatory prostaglandin E-2 [32] and proinflammatory cytokines [30], and in contrast, increased anti-inflammatory cytokines transforming growth factor-beta 1 and interleukin-10 [27, 28].

Results from the present investigation are consistent with the previous study of the 9-beam lasercomb in male AGA subjects by Leavitt et al. [8]. Both studies demonstrated a higher increase in terminal hair density with lasercomb treatment versus sham treatment, which was statistically significant, with a positive safety profile for the device. However, the current study enrolled both male and female subjects, and tested a range of laser configurations ( 8 min of treatment for the 12-beam model, $11 \mathrm{~min}$ for the 9-beam model, and 15 min for the 7-beam model, so that the three models gave similar laser dose rates per treatment), making it a more comprehensive study. While we found the lasercomb to be also efficacious in increasing terminal hair count in female subjects, we feel we cannot directly compare our results with another lasercomb study of female subjects $(n=7)$ as the baseline hair counts were too different $\left(71-307 / \mathrm{cm}^{2}\right.$ vs. $\left.8-32 / \mathrm{cm}^{2}\right)$ [9]. A recent study described the high efficacy of treating MPHL using a helmet-like low-level laser device, called TOPHAT $^{\circledR}$, in a randomized, double-blind, controlled trial [7]. While the TOPHAT $^{\circledR}$ study was for 16 weeks with treatment every other day for a total of 60 treatments versus 78 treatments in total in this lasercomb study, the laser dose rates per treatment in the TOPHAT ${ }^{\circledR}$ study were much higher (there were 215 -mW laser units). Future studies are required to optimize laser modality and treatment regimen for hair growth and maintenance.

The increase in terminal hair density per $\mathrm{cm}^{2}$ observed in our study is comparable to that observed in a 6-month randomized, investigator-blinded, controlled trial of $5 \%$ minoxidil solution in MPHL [33], but lower than that observed in 48 -week studies of $5 \%$ and $2 \%$ minoxidil topical solution in MPHL [34] and FPHL [35]. Our results in the increase in terminal hair count are comparable to $1 \mathrm{mg} /$ day finasteride treatment in some MPHL trials [36, 37], but less efficacious than longer term trials [38].

LLLT may provide a promising treatment option for patients who do not respond to either finasteride or minoxidil, and who do not want to undergo hair transplantation. Additionally, while topical minoxidil solution or foam is widely used to treat pattern hair loss and is generally well tolerated [39], the treatment needs to be applied once or twice daily, and be in contact with the scalp for at least $4 \mathrm{~h}$. Such application can be impractical for many users, leading to noncompliance and reduced efficacy. As an alternative, the lasercomb treatment is safe and easy to apply, with 8-15 min of treatment three times per week, and leaves no residue on the scalp. Such user friendliness of the lasercomb may lead to better patient compliance and improved efficacy. Future studies to modulate laser modality and treatment regimen will help optimize hair growth stimulation and maintenance by lowlevel laser.

\section{Conclusions}

In four randomized, double-blind, sham-controlled trials of MPHL and FPHL, we detected a statistically significant increase in terminal hair density after 26 weeks of lasercomb treatment compared with sham treatment. Such improvement was independent of the sex and age of the subject, and independent of the lasercomb model when similar laser dose rates were delivered. A higher percentage of lasercomb-treated subjects reported overall 
improvement of hair loss condition and thickness and fullness of hair in self-assessment, though the results did not always reach statistical significance. Increase in terminal hair count was comparable to the short-term trials of $5 \%$ minoxidil topical solution and $1 \mathrm{mg} /$ day finasteride, but less efficacious than longer term ( $\geq 1$ year) trials. Further clinical trials are needed to define the optimal duration of treatment, the duration of response, and the use of the lasercomb in other alopecia conditions.

Acknowledgments The authors would like to thank Zoe Draelos, MD (Dermatology Consulting Services, High Point), David Goldberg, MD (Skin Laser and Surgery Specialist, Hillsborough, NJ, USA; Hackensack, NJ, USA), Michael Jarratt, MD (DermaResearch, Inc., Austin, TX, USA), Abe Marcadis, MD (Palm Beach Research Center, West Palm Beach, FL, USA), and Jose Mendez, DO (International Dermatology Research, Inc., Miami, FL, USA) for their participation. Statistical analyses were contracted to Stat-Tech Services, LLC (Chapel Hill, NC, USA). Lexington International, LLC partially funded the study, and provided the treatment and sham devices and equipment (including the digital imaging system). M.R. Hamblin has received honorarium/consulting fees, and L.A. Schachner has received fees for participation from Lexington International, LLC. M. Hordinsky has received, on behalf of the Department of Dermatology at the University of Minnesota, a grant to conduct part of this study. J.J. Jimenez, T.C. Wikramanayake, W.F. Bergfeld, and J.G. Hickman have no conflicts of interest that are directly relevant to this study. M.R. Hamblin was supported by a NIH grant R01AI050875. The authors have full control of all primary data, and agree to allow the journal to review their data if requested.

Open Access This article is distributed under the terms of the Creative Commons Attribution Noncommercial License which permits any noncommercial use, distribution, and reproduction in any medium, provided the original author(s) and the source are credited.

\section{References}

1. Olsen EA, Messenger AG, Shapiro J, Bergfeld WF, Hordinsky MK, Roberts JL, et al. Evaluation and treatment of male and female pattern hair loss. J Am Acad Dermatol. 2005;52(2):301-11.

2. Hoffmann R, Happle R. Current understanding of androgenetic alopecia: Part I. Etiopathogenesis. Eur J Dermatol. 2000;10(4): 319-27.

3. Gan DC, Sinclair RD. Prevalence of male and female pattern hair loss in Maryborough. J Investig Dermatol Symp Proc. 2005;10(3):184-9.

4. McElwee KJ, Shapiro JS. Promising therapies for treating and/or preventing androgenic alopecia. Skin Therapy Lett. 2012;17(6): $1-4$.

5. Rangwala S, Rashid RM. Alopecia: a review of laser and light therapies. Dermatol Online J. 2012;18(2):3.

6. Avram MR, Rogers NE. The use of low-level light for hair growth: part I. J Cosmet Laser Ther. 2009;11(2):110-7.

7. Lanzafame RJ, Blanche RR, Bodian AB, Chiacchierini RP, Fernandez-Obregon A, Kazmirek ER. The growth of human scalp hair mediated by visible red light laser and LED sources in males. Lasers Surg Med. 2013;45(8):487-95.

8. Leavitt M, Charles G, Heyman E, Michaels D. HairMax LaserComb laser phototherapy device in the treatment of male androgenetic alopecia: a randomized, double-blind, sham device- controlled, multicentre trial. Clin Drug Investig. 2009;29(5): 283-92.

9. Satino JL, Markou M. Hair regrowth and increased hair tensile strength using the HairMax LaserComb for low-level laser therapy. Int J Cosmetic Surg Aesthetic Dermatol. 2003;5(2):113-7.

10. Norwood OT. Male pattern baldness: classification and incidence. South Med J. 1975;68(11):1359-65.

11. Ludwig E. Classification of the types of androgenetic alopecia (common baldness) occurring in the female sex. Br J Dermatol. 1977;97(3):247-54.

12. Price VH, Roberts JL, Hordinsky M, Olsen EA, Savin R, Bergfeld W, et al. Lack of efficacy of finasteride in postmenopausal women with androgenetic alopecia. J Am Acad Dermatol. 2000;43(5 Pt 1):768-76.

13. Savin R. Evaluating androgenetic alopecia in male and female patients: an improved visual method of classifying and tracking hair loss using computer-generated male and female pattern and density scales. Kalamazoo: The Upjohn Company; 1994.

14. Fitzpatrick TB, Ortonne J-P. Normal skin color and general consideration of pigmentary disorders. In: Freedberg IM, Eisen Z, Wolff K, Austen KF, Goldsmith LA, Katz SI, editors. Fitzpatrick's dermatology in general medicine. New York: McGrawHill; 2003. p. 819-26.

15. Bjordal JM, Couppe C, Chow RT, Tuner J, Ljunggren EA. A systematic review of low level laser therapy with location-specific doses for pain from chronic joint disorders. Aust J Physiother. 2003;49(2):107-16.

16. Brosseau L, Welch V, Wells G, et al. Low level laser therapy (classes I, II and III) in the treatment of rheumatoid arthritis. Cochrane Database Syst Rev. 2000(2):CD002049.

17. Cauwels RG, Martens LC. Low level laser therapy in oral mucositis: a pilot study. Eur Arch Paediatr Dent. 2011;12(2):118-23.

18. Christie A, Jamtvedt G, Dahm KT, Moe RH, Haavardsholm EA, Hagen KB. Effectiveness of nonpharmacological and nonsurgical interventions for patients with rheumatoid arthritis: an overview of systematic reviews. Phys Ther. 2007;87(12):1697-715.

19. Jamtvedt G, Dahm KT, Holm I, Flottorp S. Measuring physiotherapy performance in patients with osteoarthritis of the knee: a prospective study. BMC Health Serv Res. 2008;8:145.

20. Metelitsa AI, Green JB. Home-use laser and light devices for the skin: an update. Semin Cutan Med Surg. 2011;30(3):144-7.

21. Schubert MM, Eduardo FP, Guthrie KA, Franquin JC, Bensadoun RJ, Migliorati CA, et al. A phase III randomized double-blind placebo-controlled clinical trial to determine the efficacy of low level laser therapy for the prevention of oral mucositis in patients undergoing hematopoietic cell transplantation. Support Care Cancer. 2007;15(10):1145-54.

22. Silva GB, Mendonca EF, Bariani C, Antunes HS, Silva MA. The prevention of induced oral mucositis with low-level laser therapy in bone marrow transplantation patients: a randomized clinical trial. Photomed Laser Surg. 2011;29(1):27-31.

23. Avram MR, Leonard RT Jr, Epstein ES, Williams JL, Bauman AJ. The current role of laser/light sources in the treatment of male and female pattern hair loss. J Cosmet Laser Ther. 2007;9(1):27-8.

24. Lubart R, Eichler M, Lavi R, Friedman H, Shainberg A. Lowenergy laser irradiation promotes cellular redox activity. Photomed Laser Surg. 2005;23(1):3-9.

25. Eells JT, Wong-Riley MT, VerHoeve J, Henry M, Buchman EV, Kane MP, et al. Mitochondrial signal transduction in accelerated wound and retinal healing by near-infrared light therapy. Mitochondrion. 2004;4(5-6):559-67.

26. Pastore D, Greco M, Passarella S. Specific helium-neon laser sensitivity of the purified cytochrome c oxidase. Int J Radiat Biol. 2000;76(6):863-70.

27. Arany PR, Nayak RS, Hallikerimath S, Limaye AM, Kale AD, Kondaiah P. Activation of latent TGF-betal by low-power laser 
in vitro correlates with increased TGF-betal levels in laserenhanced oral wound healing. Wound Repair Regen. 2007; 15(6):866-74.

28. de Lima FM, Villaverde AB, Albertini R, Correa JC, Carvalho RL, Munin E, et al. Dual effect of low-level laser therapy (LLLT) on the acute lung inflammation induced by intestinal ischemia and reperfusion: action on anti- and pro-inflammatory cytokines. Lasers Surg Med. 2011;43(5):410-20.

29. Jaworsky C, Kligman AM, Murphy GF. Characterization of inflammatory infiltrates in male pattern alopecia: implications for pathogenesis. Br J Dermatol. 1992;127(3):239-46.

30. Mafra de Lima F, Villaverde AB, Salgado MA, Castro-FariaNeto HC, Munin E, Albertini R, et al. Low intensity laser therapy (LILT) in vivo acts on the neutrophils recruitment and chemokines/cytokines levels in a model of acute pulmonary inflammation induced by aerosol of lipopolysaccharide from Escherichia coli in rat. J Photochem Photobiol B. 2010;101(3): 271-8.

31. Magro CM, Rossi A, Poe J, Manhas-Bhutani S, Sadick N. The role of inflammation and immunity in the pathogenesis of androgenetic alopecia. J Drugs Dermatol. 2011;10(12):1404-11.

32. Sakurai Y, Yamaguchi M, Abiko Y. Inhibitory effect of low-level laser irradiation on LPS-stimulated prostaglandin E2 production and cyclooxygenase-2 in human gingival fibroblasts. Eur J Oral Sci. 2000;108(1):29-34.

33. Berger RS, Fu JL, Smiles KA, Turner CB, Schnell BM, Werchowski KM, et al. The effects of minoxidil, $1 \%$ pyrithione zinc and a combination of both on hair density: a randomized controlled trial. Br J Dermatol. 2003;149(2):354-62.

34. Olsen EA, Dunlap FE, Funicella T, Koperski JA, Swinehart JM, Tschen $\mathrm{EH}$, et al. A randomized clinical trial of 5\% topical minoxidil versus $2 \%$ topical minoxidil and placebo in the treatment of androgenetic alopecia in men. J Am Acad Dermatol. 2002;47(3):377-85.

35. Lucky AW, Piacquadio DJ, Ditre CM, Dunlap F, Kantor I, Pandya AG, et al. A randomized, placebo-controlled trial of 5\% and $2 \%$ topical minoxidil solutions in the treatment of female pattern hair loss. J Am Acad Dermatol. 2004;50(4):541-53.

36. Leyden J, Dunlap F, Miller B, Winters P, Lebwohl M, Hecker D, et al. Finasteride in the treatment of men with frontal male pattern hair loss. J Am Acad Dermatol. 1999;40(6 Pt 1):930-7.

37. Roberts JL, Fiedler V, Imperato-McGinley J, Whiting D, Olsen E, Shupack J, et al. Clinical dose ranging studies with finasteride, a type 2 5alpha-reductase inhibitor, in men with male pattern hair loss. J Am Acad Dermatol. 1999;41(4):555-63.

38. Kaufman KD, Olsen EA, Whiting D, Savin R, DeVillez R, Bergfeld W, et al. Finasteride in the treatment of men with androgenetic alopecia: Finasteride Male Pattern Hair Loss Study Group. J Am Acad Dermatol. 1998;39(4 Pt 1):578-89.

39. Blume-Peytavi U, Hillmann K, Dietz E, Canfield D. Garcia Bartels N. A randomized, single-blind trial of $5 \%$ minoxidil foam once daily versus $2 \%$ minoxidil solution twice daily in the treatment of androgenetic alopecia in women. J Am Acad Dermatol. 2011;65(6):1126-1134 e2. 